\title{
Editorial
}

\section{Benzodiazepines: A Valuable Tool in the Management of Cardiovascular Conditions}

\author{
Richard Balon ${ }^{a}$ Chiara Rafanelli ${ }^{b}$ Nicoletta Sonino ${ }^{c, d}$ \\ a Departments of Psychiatry and Behavioral Neurosciences and Anesthesiology, Wayne State University, \\ Detroit, MI, USA; ${ }^{b}$ Department of Psychology, University of Bologna, Bologna, Italy; ${ }^{\mathrm{C}}$ Department of \\ Statistical Sciences, University of Bologna, Bologna, Italy; ${ }^{\mathrm{d} D e p a r t m e n t}$ of Psychiatry, State University of \\ New York at Buffalo, Buffalo, NY, USA
}

The relationship between anxiety and cardiovascular disease (CVD) has been known and well-documented for decades. Though more attention has been paid to the relationship between depression and CVD, the evidence of the relationship between anxiety and CVD has been growing. For instance, Goodwin et al. [1] documented that anxiety disorders are strongly and significantly associated with CVD among adults in the USA. FrasureSmith and Lesperance [2], among others, found that, not just depression but also anxiety predicted a greater risk of major adverse cardiac events such as cardiac death, myocardial infarction, or cardiac arrest in patients with stable coronary artery disease. Less attention has been paid to the possible benefits of anxiolytic medications, such as benzodiazepines (BZs), for patients with CVD and anxiety or possible full-blown anxiety disorder.

\section{New Findings on Anxiety and CVD}

Two articles in this issue of Psychotherapy and Psychosomatics $[3,4]$ taken together suggest that this neglect of the role of anxiety and its treatment in patients with CVD may need to be reevaluated.

\section{KARGER}

() 2018 S. Karger AG, Basel

E-Mail karger@karger.com

www.karger.com/pps
In a study on patients referred by cardiologists for anxious or depressive symptoms to the Montreal Heart Institute psychosomatic outpatient clinic, Long et al. [3] looked for a specific association between CVD and anxiety disorders, and also for possible differences in the distribution of anxiety disorders in CVD patients and the general population. The diagnoses were established via unstructured interview using the DSM criteria. Out of 335 CVD patients, $30 \%$ suffered from 1 of the following anxiety disorders: panic disorder (PD)/agoraphobia $15 \%$, generalized anxiety disorder (GAD) $13 \%$, and posttraumatic stress disorder (PTSD) 3\%. The distribution of the 3 disorders in the CVD population was significantly different from that in the general population ( $p<0.0001, \chi^{2}$ goodness-of-fit test). In a multinominal logistic regression using a number of variables, having a congenital heart disease was the only variable associated with a specific anxiety disorder, GAD $(p=0.04)$. As the authors pointed out, the 3 CVD disorders they studied seem to be associated with anxiety disorders as a class. The prevalence of $\mathrm{PD} /$ agoraphobia and GAD in these patients was higher than in the general Canadian population. These findings clearly illustrate that, in addition to depressive symptomatology, we need to look for specific anxiety symptomatology in CVD patients.

Richard Balon, MD

Department of Psychiatry, Tolan Park Building 3rd Floor 3901 Chrysler Service Drive

Detroit, MI 48201 (USA)

E-Mail rbalon@wayne.edu 
In the second study, Diez-Quevedo et al. [4] looked for the possible risks of using $\mathrm{BZs}$ in patients with congestive heart failure (CHF) in terms of the impact of BZs on mortality. The study was conducted in a specialized Heart Failure Clinic in Badalona, Spain, between 2001 and 2017. The authors followed up 2,139 patients with CHF for a median of 7.96 years. Out of these patients (mostly white males), 1,052 (49.2\%) received at least a 1-month prescription for BZs (mainly alprazolam, lorazepam, diazepam, and lormetazepam). One thousand and eighty-three died during the follow-up. Interestingly, the BZ prescriptions were associated with a $15 \%$ reduction in the risk of mortality (Cox proportional hazard analysis: HR 0.84, $95 \%$ CI $0.75-0.95 ; p=0.005)$ and the significance was maintained after adjustment for a number of variables (HR 0.85, 95\% CI 0.75-0.97; $p=0.01$ ). While this study had limitations, the findings suggest that BZs could be helpful as an adjunct treatment in patients suffering from $\mathrm{CHF}$, possibly via relieving their stress, anxiety, insomnia, and/or allostatic overload [5].

\section{The Increasing Importance of Evaluating Anxiety in CVD Patients}

Depression (in various forms and as various entities) is usually the main focus of research and treatment among psychiatric comorbidities in CVD patients. Anxiety has usually been lumped together with depression as a risk factor and a treatment focus. Thus, treatment with newer antidepressants with lesser cardiac toxicity and touted efficacy for both depression and anxiety has been the preferred approach to depression and anxiety in patients with CVD. However, the results of the 2 reviewed studies $[3,4]$ suggest (a) an independent (of depression) association of anxiety and CVD, and (b) the favorable effects of medications other than newer antidepressants in patients with CVD.

Recently, De Hert et al. [6] pointed out that anxiety symptoms or disorders as well as persistent or intense stress or PTSD may also be independently associated with an increased risk of developing a particular CVD, though to a lesser degree than depression. Similarly, Celano et al. [7] noted that depression and anxiety disorders are common yet unrecognized in patients with heart failure and are linked to adverse outcomes. Thus, anxiety and anxiety disorders are becoming more frequently viewed as a possible independent risk factor in CVD patients.

\section{BZs in the Treatment of Anxiety in CVD Patients}

Treatment of anxiety/anxiety disorders associated with CVD could be as complicated and challenging as the treatment of comorbid depressive symptomatology. BZs have not always been at the forefront of treatment recommendations in this indication by various guidelines and review articles. Nevertheless, their acute and efficacious relief of anxiety in CVD patients has been recognized and documented by experienced clinicians for decades. Over 40 years ago, Lasagna [8], in his article on the use of BZs in nonpsychiatric medical practice, noted that there was "reason to believe that benzodiazepines not only possess the anxiolytic effects universally attributed to them but may also ameliorate somatic complaints affecting such systems as the cardiovascular and the gastrointestinal," in addition to conditions such as tension headache and back pain. Around the same time, Shader et al. [9] observed how physicians commonly prescribe BZs "as a part of the long-term management of certain medical conditions thought to be adversely affected by anxiety-related autonomic nervous system hyperactivity."

Several treatment studies suggested BZs' usefulness in relieving symptomatology and improving the well-being of patients with CVDs such as unstable myocardial ischemia and hypertension. A small, nonblinded, controlled study by Melsom et al. [10] demonstrated the usefulness of high-dose diazepam after myocardial infarction. In a study by Mendels et al. [11], outpatients with angina stabilized on propranolol were administered either alprazolam or placebo in a double-blind cross-over fashion. Patients receiving alprazolam together with propranolol reported more improvement of their symptoms than those on placebo. A significant reduction in the duration of silent myocardial ischemia (by 70\%) was reported in a small, double-blind, placebo-controlled study on 10 patients treated with alprazolam ( $0.5 \mathrm{mg}$ at bedtime) in addition to propranolol and transdermal nitroglycerin [12]. Based on the results of some of these studies, Williams [13] suggested that BZs could be used in the primary and secondary prevention of some medical disorders in predisposed individuals, e.g., those with type A behavior. $\mathrm{He}$ recommended that possible mechanisms of BZs, such as the reduction of stress hormone levels, the antiplatelet activation factor action, or increased parasympathetic tone, should be further investigated.

Results of some other studies suggested that BZs could also be useful as adjunct treatment of blood pressure abnormalities among patients with generalized anxiety disorder [14]. In a randomized study by Mabadeje and Ade- 
bayo [15], bromazepam was effective in controlling labile essential hypertension, although not as much as labetalol. Additive use of etizolam in patients with essential hypertension resulted in an overall improvement, a decrease in systolic and diastolic pressure, and an improvement in anxiety, fatigue, and depression in a small study by Matsuo et al. [16].

Several epidemiological studies focused on the possible risks associated with using BZs in patients with CVD. Lapane et al. [17] reported a moderate association of the risk of clinically ischemic cardiac events with $\mathrm{BZ}$ use, and a strong association of this risk with antidepressant use. The results of the study by Merlo et al. [18] suggested that the combined use of anxiolytic and hypnotic drugs and analgesics seem to be associated with an increased risk of ischemic heart disease mortality in elderly men. Then, in 2009, Krantz et al. [19] found that, while baseline anxiolytic use alone did not predict cardiovascular events, the use of antidepressants was associated with subsequent cardiovascular events in women.

Finally, in a study by Wu et al. [20], BZs were independently associated with a decreased risk of cardiac mortality and hospitalization for heart failure in patients after new myocardial infarction. Importantly, they found that patients given small-to-medium doses of had better outcomes that those given high doses (the J-curve phenomenon). They suggested that explanations for this finding could be higher comorbidity, the presence of additional systemic disease, and interference with cardiac rehabilitation.

These findings are marred by factors inherent to epidemiological studies. For instance, chronic and shortterm users are generally undifferentiated. This is actually a confounding factor, as most patients who are prescribed
BZs are short-term or intermittent users [21]. In the medically ill, there are several situations that may favor shortterm use of BZs, such as stress related to hospitalization, medical procedures, and anxiety aroused by the medical condition [22]. Another confounding factor could be lumping together all the different types of BZs, regardless of their specific characteristics [23].

Though the epidemiological studies may be frequently flawed, their results suggest that the risks associated with using BZs in CVD patients are lower than those associated with the use of antidepressants.

\section{Conclusion}

Though the results of treatment studies using BZs for the treatment of CVDs are more encouraging than the results of epidemiological studies, all these findings together demonstrate and illustrate that the endorsement by both researchers $[8,9]$ and generations of clinicians has been correct: BZs could and should be used in the treatment of anxiety/anxiety disorders, possibly as a first-line treatment, in patients with CVD, depending on the specific clinical situation. Low-to-moderate doses of adjunct BZs seem to be helpful in silent myocardial ischemia, angina, essential hypertension, and CHF, especially in patients with comorbid anxiety. It is unfortunate that BZs have not been studied in more detail in these indications.

BZs are versatile agents useful in various indications. Unlike antidepressant drugs, their use is conceived as a short-term and intermittent treatment program [9] and free of the side effects of many antidepressant drugs [24]. The time has come to reevaluate the clinical usefulness of $\mathrm{BZs}$ in the management of CVD.

\section{References}

1 Goodwin RD, Davidson KW, Keyes K: Mental disorders and cardiovascular disease among adults in the United States. J Psychiatr Res 2009;43:239-246.

2 Frasure-Smith N, Lesperance F: Depression and anxiety as predictors of 2-year cardiac events in patients with stable coronary artery disease. Arch Gen Psychiatry 2008;65:62-71.

3 Long V, Guertin M-C, Dyrda K, Benrimoh D, Brouillette J: Descriptive study of anxiety and posttraumatic stress disorder in cardiovascular disease patients: from referral to cardiopsychiatric diagnoses. Psychother Psychosom 2018, DOI: 10.1159/000491581.
4 Diez-Quevedo C, Lupon J, de Antonio M, Domingo M, Martin-Subero M, Rangil T, et al: Benzodiazepine use and long-term mortality in real-life chronic heart failure outpatients: a cohort analysis. Psychother Psychosom 2018, E-pub ahead of print.

5 Guidi J, Offidani E, Rafanelli C, Roncuzzi R, Sonino N, Fava GA: The assessment of allostatic overload in patients with congestive heart failure by clinimetric criteria. Stress Health 2016;32:63-69.

6 De Hert M, Detraux J, Vancampfort D: The intriguing relationship between coronary heart disease and mental disorders. Dialogues Clin Neurosci 2018;20:31-40.
7 Celano CM, Villegas AC, Albanese AM, Gaggin HK, Huffman JC: Depression and anxiety in heart failure: a review. Harv Rev Psychiatry 2018;26:175-184.

8 Lasagna L: The role of benzodiazepines in nonpsychiatric medical practice. Am J Psychiatry 1977;134:656-658.

9 Shader RI, Weinberger DR, Greenblatt DJ: Psychopharmacological approaches to the medically ill patient; in Karasu TB, Steinmuller RI (eds): Psychotherapeutics in Medicine. New York, Grune and Stratton, 1978, pp 117-155.
Benzodiazepines in Cardiovascular Disease
Psychother Psychosom 2018;87:327-330 DOI: $10.1159 / 000493015$ 
10 Melsom M, Andreassen H, Melsom H, Hansen T, Grendahl H, Hillestad LK: Diazepam in acute myocardial infarction. Br Heart J 1976; 38:804-810.

11 Mendels J, Chernoff RW, Blatt M: Alprazolam as an adjunct to propranolol in anxious outpatients with stable angina pectoris. J Clin Psychiatry 1986;47:8-11.

12 Shell WE, Swan HJ: Treatment of silent myocardial ischemia with transdermal nitroglycerin added to beta-blockers and alprazolam. Cardiol Clin 1986;4:697-704.

13 Williams RB Jr: Do benzodiazepines have a role in the prevention or treatment of coronary heart disease and other major medical disorders? J Psychiatr Res 1990;24:51-56.

14 Fontaine R, Boisvert D: Psychophysiological disorders in anxious patients: hypertension and hypotension. Psychother Psychosom 1982;38:165-172.

15 Mabadeje AF, Adebayo GI: Comparative effects of labetalol and bromazepam on ambulatory blood pressure of Nigerians with labile and stress hypertension. Clin Exp Hypertens A 1989;11(suppl 1):441-447.
16 Matsuo H, Watanabe S, Ishiguro A, Arai M, Sugiyama A, Matsuno Y, et al: The efficacy of addictive use of etizolam in patients with essential hypertension and unspecified complaints. Int J Clin Pharmacol Ther Toxicol 1992;30:51-56.

17 Lapane KL, Zierler S, Lasater TM, Barbour MM, Carleto R, Hume AL: Is the use of psychotropic drugs associated with increased risk of ischemic heart disease? Epidemiology 1995;6:376-381.

18 Merlo J, Hedblad B, Ogren M, Ranstam J, Ostergren PO, Ekendal A, et al: Increased risk of ischaemic heart disease mortality in elderly men using anxiolytics-hypnotics and analgesics. Results of the 10-year follow-up of the prospective study "Men born in 1914," Malmo, Sweden. Eur J Clin Pharmacol 1996;49: 261-265.

19 Krantz DS, Whittaker KS, Francis JL, Rutledge T, Johnson BD, Barrow G, et al: Psychotropic medication use and risk of adverse cardiovascular events in women with suspected coronary artery disease: outcomes from the Women's Ischemia Syndrome Evaluation (WISE) study. Heart 2009;95:1901-1906.
20 Wu C-K, Huang Y-T, Lee J-K, Jimmy Juang J-M, Tsai C-T, Lai L-P, et al: Anti-anxiety drugs use and cardiovascular outcomes in patients with myocardial infarction: a nationwide assessment. Atherosclerosis 2014;235: 496-502.

21 Cloos JM, Bocquet V, Rolland-Portal I, Koch P, Chouinard G: Hypnotics and triazolobenzodiazepines-best predictors of high-dose benzodiazepine use: results from the Luxembourg National Health Insurance Registry. Psychother Psychosom 2015;84:273-283.

22 Fava GA, Cosci F, Sonino N: Current psychosomatic practice. Psychother Psychosom 2017;86:13-30.

23 Cosci F, Guidi J, Balon R, Fava GA: Clinical methodology matters in epidemiology: not all benzodiazepines are the same. Psychother Psychosom 2015;84:262-264.

24 Offidani E, Guidi J, Tomba E, Fava GA: Efficacy and tolerability of benzodiazepines versus antidepressants in anxiety disorders: a systematic review and meta-analysis. Psychother Psychosom 2013;82:355-362. 